\title{
COVID-19 and the role of chronic inflammation in patients with obesity
}

\author{
Sonja Chiappetta $\mathbb{1}^{1} \cdot$ Arya M. Sharma ${ }^{2} \cdot$ Vincenzo Bottino $^{1} \cdot$ Christine Stier $^{3,4}$
}

Received: 21 March 2020 / Revised: 28 April 2020 / Accepted: 5 May 2020 / Published online: 14 May 2020

(c) Springer Nature Limited 2020

\begin{abstract}
Coronavirus disease 2019 (COVID-19) and the risk of severe acute respiratory syndrome coronavirus 2 (SARS-CoV-2) poses a particular risk to people living with preexisting conditions that impair immune response or amplify pro-inflammatory response. Low-grade chronic systemic inflammation, common in people with obesity, is associated with the development of atherosclerosis, type 2 diabetes, and hypertension, well known comorbidities that adversely affect the outcomes of patients with COVID-19. Risk stratification based on the Edmonton Obesity Staging System (EOSS), which classifies obesity based on the presence of medical, mental, and/or functional complications rather than on body mass index (BMI), has been shown to be a better predictor of all-cause mortality and it may well be that EOSS stages may better describe the risk of hyperinflammation in patients with COVID-19 infection. Analyzing a group of metabolic ill patients with obesity (EOSS 2 and 3), we found an increased interleukin-6 and linear regression analysis showed a positive correlation with C-reactive protein (CRP) $(p=0.014)$ and waist-to-hip-ratio (WHR) $(p=0.031)$. Physicians should be aware of these findings in patients with COVID-19 infection. Early identification of possible hyperinflammation could be fundamental and should guide decision making regarding hospitalization, early respiratory support, and therapy with immunosuppression to improve mortality.
\end{abstract}

The current pandemic of COVID-19 and the risk of SARS$\mathrm{CoV}-2$ poses a particular risk to people living with preexisting conditions that impair immune response or amplify pro-inflammatory response. Given the coexistence of a world-wide epidemic of obesity, which in some Western countries affects up to a $40 \%$ of the general population [1], it is prudent to consider the impact that COVID-19 infection can have on people living with obesity. Notably, elevated body mass index (BMI) and obesity were predictors of poor outcomes and fatalities due to H1N1 [2]. Moreover, observations from Wuhan show that hypertension (30\%),

Sonja Chiappetta

drschiappetta@gmail.com

1 Department of Obesity and Metabolic Surgery, Ospedale Evangelico Betania, Naples, Italy

2 Division of Endocrinology, Department of Medicine, University of Alberta, Edmonton, AB, Canada

3 Obesity Center NRW, Sana Kliniken Germany, Huerth, Germany

4 Clinic for General, Visceral and Transplant Surgery, University Hospital Aachen, Aachen, Germany diabetes (22\%), and coronary artery disease (22\%), all of which are more common in people living with obesity, were the most common comorbidities in patients requiring hospitalisation [3, 4]. Poorly, in the Chinese studies obesity rates were not reported.

Chronic inflammation, which accompanies obesity and metabolic syndrome, leads to abnormal cytokine production and increased acute phase reactants. Patients with obesity have a higher activity of nuclear transcription factor kappa $\mathrm{B}$ and a higher ribonucleic acid expression of proinflammatory cytokines, such as tumor necrosis factor- $\alpha$, interleukin-1, and interleukin-6 (IL-6), crucial elements in the pathogenesis of metabolic syndrome [5]. The innate immune response in patients with obesity is altered and leads to an altered first line of defense, increased inflammatory response, and abnormal T-cell response [6]. Furthermore, the key role of IL-6 in mediating the acute phase response and its value as a prognostic biomarker in sepsis and various acute organ injuries has been extensively investigated in clinical and experimental studies [7]. The pro-inflammatory role of IL-6 is often described in the pathogenesis of lung diseases, especially in asthma [8] and in patients with acute respiratory distress syndrome [7]. 
Plasma and/or broncho-alveolar levels of IL-6 have been identified as early biomarkers of lung injury [7]. IL-6 acts on hepatocytes to induce expression of CRP as a part of the acute phase response and may also play a central role for the development and progression of liver disease [9].

In patients affected by COVID-19, it seems that agedependent defects in T-cell and B-cell function and the excess production of type 2 cytokines could lead to a deficiency in control of viral replication and more prolonged pro-inflammatory responses, potentially leading to poor outcome [3]. Different risk factors include sex and smoking [10], hypertension and diabetes mellitus, and the treatment with angiotensin-converting enzyme 2-increasing drugs [11]. The potential risk factors for poor prognosis in early stage include older age, high sequential organ failure assessment score, and d-dimer greater than $1 \mu \mathrm{g} / \mathrm{l}$ [3]. Lowgrade systemic inflammation, as is common in people living with obesity, is associated with the development of insulin resistance, dyslipidemia, atherosclerosis, type 2 diabetes, hypertension, and asthma [8], well known comorbidities that adversely affect the outcomes of patients with COVID19 [12-15]. The role of the liver, as a key organ that performs immune response against pathogens and the high incidence of nonalcoholic steatohepatitis in patients with obesity and chronic inflammation, has still to be cleared in patients affected by COVID-19.

In addition to a possible role of obesity related metaflammation, or metabolically triggered inflammation in promoting health risks related to COVID-19 and SARSCoV-2, patients with severe obesity also present important management challenges with regard to ventilation support, vascular access, and decubital skin breakdown [16].

It may be important to also note that BMI may not be the best indicator of obesity related morbidity or mortality. Thus, for example, risk stratification based on the EOSS [17], which classifies obesity in five stages based on the presence of medical, mental, and/or functional complications rather than on BMI has been shown to be a better predictor of all-cause mortality in patients with obesity [18] and adverse outcomes in patients undergoing bariatric surgery [19]. It may well be that EOSS stages may better describe the risk associated with COVID-19 infections in patients with obesity than BMI alone.

Due to the actual pandemic and the discussion regarding IL-6, we reanalyzed a subpopulation of patients (Sana Klinikum Offenbach, Germany), who participated in our recent inflammation study [20, 21] and underwent measurement of IL-6 (pg/ml) in the standard preoperative work up $(n=33)$. The study aimed to compare different bariatric procedures pertaining with their effects on inflammation markers in the short and long term and was conducted in accordance with the principles of the Declaration of Helsinki. Ethical permission was obtained from the local ethics
Table 1 Baseline demographic data of a subpopulation of patients (n =33), who participated in the inflammation study [20, 21] and underwent measurement of IL- $6(\mathrm{pg} / \mathrm{ml})$ in the standard preoperative work up.

\begin{tabular}{ll}
\hline & Values \\
\hline Age (in years) & $44.51 \pm 11.08(24-61)$ \\
Waist-to-hip ratio & $0.98 \pm 0.15(0.77-1.5)$ \\
BMI $\left(\mathrm{kg} / \mathrm{m}^{2}\right)$ & $61.94 \pm 12.3(35-83.2)$ \\
Interleukin-6 $(\mathrm{pg} / \mathrm{ml})$ & $9.21 \pm 4.85(1.2-21.86)$ \\
CRP $(\mathrm{mg} / \mathrm{l})$ & $17.76 \pm 22.91(4-99)$ \\
\hline
\end{tabular}

Standard values of C-reactive protein (CRP) and interleukin-6 (IL-6) are $<5 \mathrm{mg} / \mathrm{l}$ and $<7 \mathrm{pg} / \mathrm{ml}$.

committee (Landesaerztekammer Hessen, Germany, reference number FF 145/2015). All participants provided written informed consent for data sharing. The National Clinical Trials number was NCT02697695.

All of the patients were scored EOSS $2(60.4 \%)$ and 3 (39.4\%). Demographic data and laboratory values are listed in Table 1. Mean preoperative IL-6 and CRP was elevated with a mean of 9.21 and $17.76 \mathrm{mg} / \mathrm{l}$ in this subpopulation of patients with the presence of comorbidities (EOSS 2) and end-organ damage (EOSS 3). In the multiple linear regression model a significant regression equation was found regarding WHR, BMI, age, and CRP $(F(4,28)=$ 4.072, $p=0.0010$ ) with an $R^{2}$ of 0.368. A statistically significant beta value was seen for WHR (beta value = $0.360, p=0.031$ ) and CRP (beta value $=0.446, p=0.014$ ). No statistically significant correlation was found between BMI and IL-6 ( $p=0.565)$, BMI and WHR $(p=0.238)$, and BMI and CRP $(p=0.183)$. A significant correlation was found between BMI and age $(p=0.048)$.

Recent publications discuss the role of immunosuppression as a treatment option in patients with COVID-19 infection, since accumulating evidence suggests that a subgroup of patients with severe COVID-19 might have a hyperinflammation and cytokine storm syndrome [22]. Interestingly, organomegaly (hepatomegaly and/or splenomegaly), triglycerides, and serum aspartate aminotransferase were identified as risk factors for the development of hyperinflammatory syndrome, well known factors that are present in patients with metabolic syndrome (EOSS $\geq 2$ ).

The higher basal rate of CRP and IL-6 in our study cohort of patients with obesity and EOSS $\geq 2$ confirms the impaired immune response in these patients. Importantly, no correlation was found to BMI, but a correlation was found to WHR. These results might explain the high risk of poor outcomes of SARS-CoV-2 in metabolic ill patients.

In conclusion, further studies are necessary to confirm the role of chronic inflammation and IL- 6 due to obesity in the pathogenesis of COVID-19 and SARS-CoV-2. Physicians should be aware of the risk of hyperinflammation in 
patients with COVID-19 infection. EOSS stages may better describe the risk of hyperinflammation in patients with COVID-19 infection than BMI. Early identification of possible hyperinflammation could be fundamental and should guide decision-making regarding hospitalization, early respiratory support, and therapy with immunosuppression to improve mortality.

Author contributions All authors performed substantial contributions to conception and design of the article and to acquisition, analysis, and interpretation of data. All authors reviewed the manuscript for important intellectual content and approved the final version for publication. All authors agree to be accountable for all aspects of the work in ensuring that questions related to the accuracy or integrity of any part of the work are appropriately investigated and resolved.

\section{Compliance with ethical standards}

Conflict of interest The authors declare that they have no conflict of interest.

Publisher's note Springer Nature remains neutral with regard to jurisdictional claims in published maps and institutional affiliations.

\section{References}

1. Hales CM, Carroll MD, Fryar CD, Ogden CL. Prevalence of obesity among adults and youth: United States, 2015-2016. NCHS Data Brief. 2017;288:1-8.

2. Poulakou G, Perez M, Rello J. Severe acute respiratory infections in the postpandemic era of H1N1. Curr Opin Crit Care. 2012;18:441-50.

3. Zhou F, Yu T, Du R, Fan G, Liu Y, Liu Z. et al. Clinical course and risk factors for mortality of adult inpatients with COVID-19 in Wuhan, China: a retrospective cohort study. Lancet. 2020;395: 1054-62.

4. Fang L, Karakiulakis G, Roth M. Are patients with hypertension and diabetes mellitus at increased risk for COVID-19 infection? Lancet Respir Med. 2020;8:e21.

5. Hotamisligil GS. Inflammation and metabolic disorders. Nature. 2006;444:860-7.

6. Frydrych LM, Bian G, O'Lone DE, Ward PA, Delano MJ. Obesity and type 2 diabetes mellitus drive immune dysfunction, infection development, and sepsis mortality. J Leukoc Biol. 2018; 104:525-34.
7. Voiriot G, Razazi K, Amsellem V, Tran Van Nhieu J, Abid S, Adnot S, et al. Interleukin-6 displays lung anti-inflammatory properties and exerts protective hemodynamic effects in a doublehit murine acute lung injury. Respir Res. 2017;18:64.

8. Peters MC, McGrath KW, Hawkins GA, Hastie AT, Levy BD, Israel E, et al. Plasma interleukin-6 concentrations, metabolic dysfunction, and asthma severity: a cross-sectional analysis of two cohorts. Lancet Respir Med. 2016;4:574-84.

9. Gudowska-Sawczuk M, Wrona A, Gruszewska E, Cylwik B, Panasiuk A, Flisiak R, et al. Serum level of interleukin-6 (IL-6) and N-terminal propeptide of procollagen type I (PINP) in patients with liver diseases. Scand J Clin Lab Investig. 2018;78:125-30.

10. Cai H. Sex difference and smoking predisposition in patients with COVID-19. Lancet Respir Med. 2020;8:e20

11. Fang L, Karakiulakis G, Roth M. Are patients with hypertension and diabetes mellitus at increased risk for COVID-19 infection? Lancet. Respir Med.2020;8:e21

12. Wang T, Du Z, Zhu F, Cao Z, An Y, Gao Y. Comorbidities and multi-organ injuries in the treatment of COVID-19. Lancet. 2020;395:e52

13. Yang X, Yu Y, Xu J, Shu H, Xia J, Liu H. et al. Clinical course and outcomes of critically ill patients with SARS-CoV-2 pneumonia in Wuhan, China: a single-centered, retrospective, observational study. Lancet Respir Med.2020;8:475-81.

14. Guan WJ, Ni ZY, Hu Y, Liang WH, Ou CQ, He JX. et al. Clinical characteristics of Coronavirus disease 2019 in China. N Engl J Med. 2019;382:1708-20.

15. Zhang JJ, Dong X, Cao YY, Yuan YD, Yang YB, Yan YQ. et al. Clinical characteristics of 140 patients infected with SARS-CoV-2 in Wuhan, China. Allergy. 2020; https://doi.org/10.1111/all.14238.

16. Selim BJ, Ramar K, Surani S. Obesity in the intensive care unit: risks and complications. Hosp Pract. 2016;44:146-56.

17. Sharma AM, Kushner RF. A proposed clinical staging system for obesity. Int J Obes. 2009;33:289-95.

18. Kuk JL, Ardern CI, Church TS, Sharma AM, Padwal R, Sui X, et al. Edmonton obesity staging system: association with weight history and mortality risk. Appl Physiol Nutr Metab. 2011;36:570-6.

19. Chiappetta S, Stier C, Squillante S, Theodoridou S, Weiner RA. The importance of the Edmonton obesity staging system in predicting postoperative outcome and 30-day mortality after metabolic surgery. Surg Obes Relat Dis. 2016;12:1847-55.

20. Chiappetta S, Jamadar P, Stier C, Bottino V, Weiner RA, Runkel $\mathrm{N}$. The role of C-reactive protein after surgery for obesity and metabolic disorders. Surg Obes Relat Dis. 2020;16:99-108.

21. Chiappetta S, Schaack HM, Wolnerhannsen B, Stier C, Squillante $\mathrm{S}$, Weiner RA. The impact of obesity and metabolic surgery on chronic inflammation. Obes Surg. 2018;28:3028-40.

22. Mehta P, McAuley DF, Brown M, Sanchez E, Tattersall RS, Manson JJ. COVID-19: consider cytokine storm syndromes and immunosuppression. Lancet. 2020;395:1033-4. 